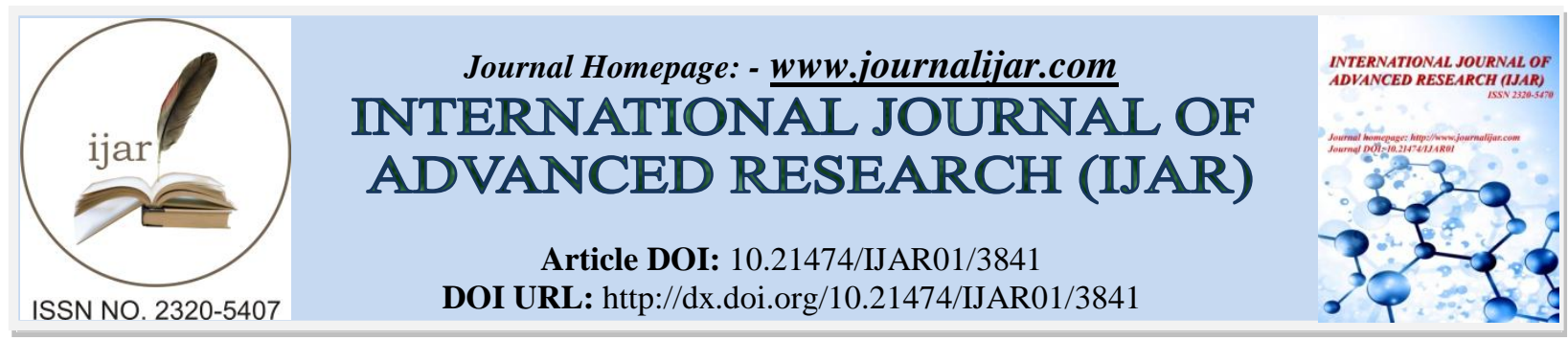

RESEARCH ARTICLE

\title{
ODIA LANGUAGE TO SIGN LANGUAGE MACHINE TRANSLATION SYSTEM: A FACE-TUBE FOR AUDIBLE IMPAIRED PEOPLE.
}

Rudranarayan Mohapatra and Anadi Charan Gan.

P.G. Department of Odia, Utkal University, Vanivihar, Bhubaneswar - 71004

\section{Manuscript Info}

Manuscript History

Received: 01 February 2017

Final Accepted: 08 March 2017

Published: April 2017

Key words:-

Indian Sign Language (ISL), Dynamic Image Database for Facial gesture (DIDFG), Face Gesture Recognition (FGR), Machine Translation (MT)

\begin{abstract}
At present, due to user-to user social experience content provider, the YouTube is major platform and buzz for disseminating multimedia information. However, looking to the Audible impaired people, the constraint came out to establishing communication with normal language to sign language irrespective of availability of its subscription graph, comment graph, and video content corpus. The time has come to establish a bridge between these social sights to Audible challenged people effectively. Therefore, here is the small step to prepare a prototype face-tube to overcome the language barrier. In this paper, we have tried to provide a diagrammatic solution for Odia language to Sign language translation process for future communication process. Here, a step is taken to Recognize Subtle Facial Expression in an effective language communication as an input and addition of Speech to Text conversion system. Again Translating the text to Sign language and converting the same to Multimedia Symbols how can coherently develop a face-tube is being explained.
\end{abstract}

Copy Right, IJAR, 2017,. All rights reserved.

\section{Introduction:-}

Developing translation systems for hearing impaired i.e. well equipped with comprehensive linguistics, computer graphics and agent knowledge has led to the development of increasingly sophisticated and specialized communicative software are very important. However, for past few years, we have seen an ever increasing development of machine translation systems for translating between major spoken natural languages, the research towards translation of Indian languages like Odia to Sign language is virtually ignored or not satisfactory by the machine translation community. To overcome this barrier, here we are aim to design a prototype Face-tube for Audible Impaired people that could translate Odia Language to sign language as per the users understanding prospective.

\section{Aim and Objective:-}

Communication between the audible impaired from different countries or community is a problem. From the Eighties, analysis and processing of sign languages among researchers is an open challenge. Initially the sigh language system was designed and developed for communication between hearing and deaf people and/or intraaudible challenged people. Sign languages are made up of both manuals components and non-manual components such as gaze, facial expressions and emotions.

Therefore, our aim is to develop a Face tube in Indian languages that could interact among Audible Impaired people to make the distant communication much easier. The software would works in between the Speech to Sign language 
Machine Translation Environment where speech would converted into source text and the same later processed by a special translator into sign language. The sign language itself refers to a Dictionary of Deaf people and transforms the text into Multimedia animated objects.

\section{Odia Language \& Sign language:-}

Odia is among 22 scheduled languages recognized by the constitution of India belong to the eastern group of the Indo-Aryan languages. The earliest established written texts in Odia language are approximately thousand years old. It is spoken predominantly in the Indian state of Orissa by and large of 35 million people.

In other way, sign language is a visual language which talks with help of hand movements, body parts and facial expressions. Hundreds of sign languages are used around the world. Japanese Sign Language (Nihon Shuwa, JSL), British Sign Language (BSL) and Turkish Sign Language (Türk İşaret Dili, TID) are out of them. As per Census 2011, on an average of total population of India 18 percent of people with disabilities have a hearing impairment. Compared to this, we do not have a composite Indian Sign Language (INSL). Studies also revealed that, one out of every five deaf people in the world is from India.

\section{Sign Language MT System:-}

TESSA: A Speech-To-British Sign Language translation system that aims to provide a communication aid between a deaf person and a Post Office clerk. INGIT is a Hindi-To-Indian Sign Language (ISL) Machine Translation system has been built for the railway reservation domain (Kar et. al, 2006). INGIT is based on Hybrid formulaic grammar approach.

\section{Except the above some of the patents in this area are:-}

- Digital Sign language translator is an US Patent by Barbara Ander, Sidney Ander having Publication Number is US 8566077 B2 and Publication date is 22 Oct 2013

- $\quad$ Sign language translator Patent by Google having Publication number is US20020152077 A1 and Publication date is Oct 17, 2002

- Language translation apparatus and method using context-based translation models by IBM having US 5510981 A and Publication date is 23 Apr 1996

\section{Features of the System:-}

The better system is that could act as interpreters between vocal and hearing-impaired people and would facilitate the life of deaf and integrate them in the competitive society. Such systems would perform bidirectional translation of sign language and spoken language in Indian language perspective. The system can:
a) Recognize Subtle Facial Expression
b) Convert Speech to Text
c) Translate Text to Sign language
d) Convert Sign language to Multimedia Symbols

Therefore, the Subtle Face Expression and language speech is going to be an input to this system. The speech is going to be converted to Text form followed by text to sign language will interred one or more translated algorithms for its translation.

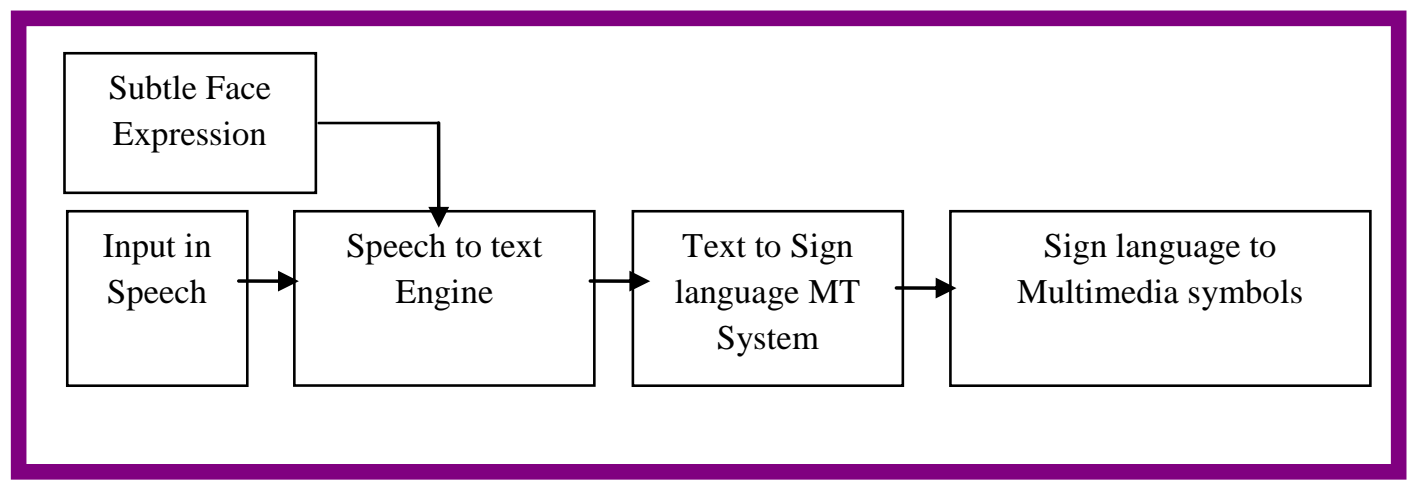

Figure 1:- Overall Architecture of the System 


\section{Expected subsystems:-}

1. Gesture Recognizer

2. Speech-to-Text Conversion System

3. Text to Sign language MT System

4. Sign Language to Multimedia Animated Object Creator

\section{System Description:-}

Therefore, the proposed prototype diagrammatic model is to be a surveillance tool in Indian languages that could interact among Audible Impaired people to make the distant communication much easier. The software would works in between the Speech to Sign language Machine Translation Environment where speech would converted into source text and the same later processed by a special translator into sign language. The sign language itself refers to a Dictionary of Deaf people and transforms the text into Multimedia animated objects.

\section{Sub-components of the system are:-}

1. Face Gesture Recognition:

$>$ Pre-processing of Face Recognition

$>$ Facial Feature Detection

$>$ Facial Gesture Extraction Representation

2. Audio-Visual Speech Synthesis

3. Text to Sign Language MT System

4. Text-to-Sign-Symbol Conversion System

$>$ Pre-process \& Tokenization

$>$ Image Database

$>$ Search Memory for matching video

\section{Detailed descriptions of the modules are as follows:-}

Face Gesture Recognition: Face Gesture recognizer would follow the vision-based method initially Appearance modeling and then 3D face/neck modeling. Initially the system considers 2D video sequences of face 7 neck as the input which would process through two major steps: face gesture region detection $\&$ face gesture recognition.

Pre-processing of Face Recognition: It aims to detect the face gesture region try to fix the valid frames and locate the face region from rest of the images. Detect skin region from the rest of the image by using color. Then transform the detection results in to the gray scale images.

Facial Feature Detection: This module would follow the multi-phase multi-detector processing of an input frontalview face image sequence. This phase follow two phases of for detection of prominent facial features. In first phase the color-based segmentation of the face would be segmented from an input frame of largest connected image component. In second phase, based upon spatially sample the contour of a certain permanent facial feature the system one or more facial-feature detectors to the pertinent Region of Interest that to be analyzed with the Dynamic Image Database for Facial gesture (DIDFG) which is the collection of facial gesture with rule based approach

Facial Gesture Extraction Representation: The contours of the facial features, generated by the facial feature detection method, would be utilized for further analysis of facial gestures to provide the input for Audio-visual speech synthesis system.

Audio-Visual Speech Synthesis: In the degradation of the auditory signal condition, speech-recognition is always a constraint. To overcome these difficulties the above mention gesture recognition features (lip-motion) would play an important role. So this module would play the role to cater visual speech onset or offset detection.

Text to Sign Language MT System: The Text to Sign language MT Module will be overall responsible for Preprocess, i.e. comprising of Corpus Creation \& Tokenization, If from its shallow parsing, the desired word available from one or more image database. In this connection the memory of matching video behaves as a deep level parser to search the data from image database. If the video found the translated output is to be displaced by appropriate syntactic information. Whereas if the video are not found it is required to break the words into images for fetching the desired image from image database. The details of sign language MT System is at Fig No. 2. 


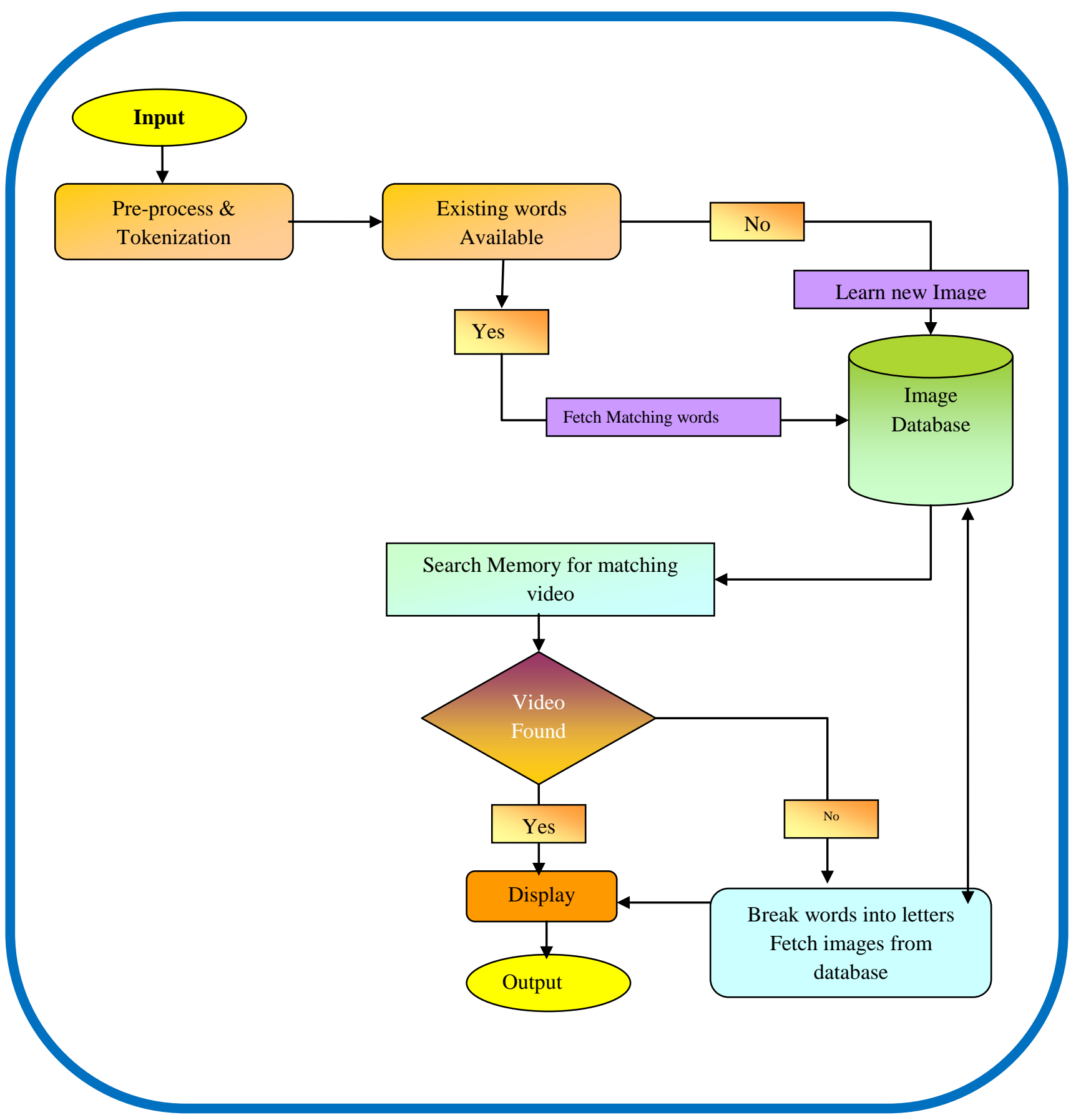

Figure 2:- Architecture of Sign Language MT System

Text-to-Sign-Symbol Conversion System: The module would capable of recognizing sign-language symbols can be used as a means of one-way communication from deaf to vocal people. This module can follow image dictionary method for the detection of sign language symbols. 
For the development of this system, "a semantic representation of the sign language would be considered. As a convention, the meaning of the sign is written as the upper case stem form of the corresponding word in a spoken language, with additional spatial information and facial expression added." ${ }^{1}$

\section{Conclusion:-}

Like any other machine translation system, parsing method influences the translation phase to a large degree, the proposed system may show its own advantages and disadvantages. In addition, as the sign languages are visualspatial rather than written languages, some unique problems may arise both its parsing and generation phases looking to the language specific constraint. With this, problems like the construction of the signing space and the generation of facial expressions and body movement. Typically, successful translation in this regard would require semantic and discourse analysis, with varying degrees of success. Further its implementation and discourse will open the new solutions to the future.

\section{Reference:-}

- Liwei Zhao, Karin Kipper,William Schuler \& et.al, University of Pennsylvania,, "A Machine Translation System from English to American Sign Language", University of Pennsylvania ScholarlyCommons, October 2000

- Mirjam Wattenhofer, Google Zurich; Roger Wattenhofer, ETH Zurich; Zack Zhu, ETH Zurich,"The YouTube Social Network",

https://static.googleusercontent.com/media/research.google.com/en//pubs/archive/37738.p

- Achraf Othman and Mohamed Jemni, "Statistical Sign Language Machine Translation: from English written text to American Sign Language Gloss", IJCSI International Journal of Computer Science Issues, Vol. 8, Issue 5, No 3, September 2011, ISSN (Online): 1694-0814, www.IJCSI.org

- Sara Morrissey and et.al, Andy Way School of Computing Dublin City University, Ireland, Page Number:2 http://www.mt-archive.info/MTS-2007-Morrissey.pdf

- Tirthankar Dasgupta, Sandipan Dandpat, Anupam Bas, "Prototype Machine Translation System From Text-ToIndian Sign Language", Proceedings of the IJCNLP-08 Workshop on NLP for Less Privileged Languages, pages 19-26, Hyderabad, India, January 2008, Asian Federation of Natural Language Processing

- LYNETTE VAN ZIJL and ANDRIES COMBRINK, "The South African Sign Language Machine Translation Project: Issues on Non-manual Sign Generation", Stellenbosch University, Proceedings of SAICSIT 2006, Pages 1-8, https://pdfs.semanticscholar.org/8fc4/f587dc788464e6641f21bfab6b21c5918e82.pdf

\footnotetext{
${ }^{1}$ Sara Morrissey and et.al, Andy Way School of Computing Dublin City University, Ireland, Page Number:2 http://www.mt-archive.info/MTS-2007-Morrissey.pdf
} 